\title{
Study of the Brainstem, Particularly the Arcuate Nucleus, in Sudden Infant Death Syndrome (SIDS) and Sudden Intrauterine Unexplained Death (SIUD)
}

\author{
Luigi Matturri, MD, PhD, Giulia Ottaviani, MD, Graziella Alfonsi, BD, Marina Crippa, BD, \\ Lino Rossi, MD, and Anna M. Lavezzi, MD
}

\begin{abstract}
Complete examination of the brainstem involves transverse serial $5-\mu \mathrm{m}$ sections made throughout the entire brainstem. The number of serial sections varies from 360 in sudden intrauterine unexplained death (SIUD) to 600 in term fetuses to over 1400 sections in sudden infant death syndrome (SIDS) victims. The procedure is not applicable in all histopathological laboratories, owing to the need for additional technical personnel. The simplified procedure allows a remarkable reduction of the number of sections. The brainstem is divided into 3 blocks. The first, cranial block, extends from the border between the medulla oblongata and pons up to the upper pole of the olivary nucleus. The second, intermediate block, corresponding to the submedian area of the inferior olivary nucleus, has as reference point the obex and extends 2 to $3 \mathrm{~mm}$ above and below the obex itself. The third, caudal block, includes the lower pole of the inferior olivary nucleus and the lower adjacent area of the medulla oblongata. Examinations of the brainstems from 106 SIDS victims, 30 controls, and 51 stillborns underlined a remarkable variability, particularly of the arcuate nucleus. The simplified examination of the brainstem makes it possible to evaluate the structures, examining 3 specific levels, defined by morphologic reference points.
\end{abstract}

Key Words: SIDS, SIUD, brainstem, arcuate nucleus

(Am J Forensic Med Pathol 2004;25: 44-48)

$\mathrm{T}$ he sudden infant death syndrome (SIDS) is defined as "the sudden death of an infant under one year of age which remains unexplained after a thorough case investigation, including performance of a complete autopsy, examination of the death scene, and review of the clinical history." It

Manuscript received March 14, 2003; accepted October 31, 2003.

From the Institute of Pathology, University of Milan, Italy.

Address correspondence and reprints request to Luigi Matturri, MD, Chairman, Institute of Pathology, University of Milan, Via della Commenda, 19, 20122 Milan, Italy. E-mail: luigi.matturri@unimi.it Copyright (C) 2004 by Lippincott Williams \& Wilkins

ISSN: 0195-7910/04/2501-0044

DOI: 10.1097/01.paf.0000113813.83779.21 is the most common form of death among babies in the first year of life.

In the last few years, there has been a $50 \%$ decline in the SIDS rate due to the identification of specific environmental risk factors, particularly the prone position in the crib, and maternal smoking. ${ }^{2}$ However, the SIDS pathogenesis is still largely unknown, and its persistent high frequency has led to continuous research to uncover the anatomopathologic basis.

The better-accepted theories (respiratory apnea, cardiac arrhythmias, visceral dyskinetic-upper digestive alterations) attribute a role to the autonomic nervous system. ${ }^{3,4}$

Sudden intrauterine unexplained death (SIUD) is defined as late unexplained fetal death before the complete expulsion of the fetus from the mother., ${ }^{3,5,6}$ Advances in maternal and fetal care have produced a significant reduction in perinatal mortality but have not changed the prevalence of SIUD, whose etiology remains largely unexplained.

Recent observations have identified, both in SIDS and SIUD victims, frequent developmental abnormalities in the brainstem, particularly in the arcuate nucleus (ARCn). ${ }^{7-12}$ The ARCn is an important cardiorespiratory center of the ventral medullary surface, characterized by a great morphologic variability. ${ }^{8,11}$ ARCn hypoplasia, detected in over 30\% of SIDS and SIUD cases, ${ }^{10-12}$ is of great interest, particularly because of its frequency and its pathogenic implications. Sometimes it is associated with alterations in other brainstem structures, particularly the respiratory reticular formation, the tractus solitarius nucleus, the muscarinic cholinergic receptors, etc. Notably, and likely because of its functional consequences, ARCn hypoplasia can be unilateral and when bilateral may involve only an area of the ARCn. ${ }^{10-12}$ All these findings need further studies on larger series involving complete, standardized examination of the brainstem on serial sections.

The aim of the present work is to describe the anatomopathologic techniques that can be adopted for the study of the ARCn, as well as of the other respiratory, cardiovascular 
and arousal coordinating structures of the brainstem. A simplified procedure for the routine study of medullary structures, both in fetuses after the 25th week and in infants, will also be presented, applicable in all histopathological laboratories. Preliminary findings have been reported in abstract form. ${ }^{13}$

\section{MATERIALS AND METHODS}

This study was performed on brainstems of 106 SIDS victims (ranging in age from 0 to 365 days), 30 deceased control infants (age-matched), and 51 stillborns after the 25th week of gestation.

The procedure for the complete examination of the brainstem, after fixation in $10 \%$ phosphate-buffered formalin for 3-4 days, is the following:

- in the stillborn, the brainstem specimen, including the lower third of the midbrain cranially and the medulla oblongata caudally sectioned some millimeters distally to the lower pole of the inferior olivary nucleus, is entirely processed;

- in SIDS victims, because of the greater size, the brainstem is divided into 2 parts. 1) The first specimen comprises the medulla oblongata. The upper cut is conducted some millimeters proximally to the border between the medulla oblongata and the pons; the lower cut is conducted some millimeters below the lower pole of the olive; 2) the second specimen comprises the pons and the lower third portion of the midbrain.

- In all cases it is important to avoid removing the leptomeninges for fear of tearing, to avoid discontinuity of the ventral medullary surface.

All specimens, after fixation, are dehydrated in ethanol at increasing concentrations and included in paraffin with a fusion point at $56^{\circ} \mathrm{C}$ to $58^{\circ} \mathrm{C}$. These passages in ethanol and then in paraffin, to optimize the results, are done manually, varying the times according to the specimen size. The number of serial sections throughout the entire brainstem varies in relation to the age of the victim analyzed. In the fetus, from the 25 th week of gestation, the average number of sections is 360 , while in the fetus at term and/or in the newborn, it is 600 . In SIDS victims of 3 to 4 months or over 6 months, 900 and 1400 sections are made, respectively. This procedure allows a detailed evaluation, apart from the ARCn, of all the other brainstem nuclei.

To apply the appropriate histologic staining (hematoxylin/eosin, Klüver-Barrera, and trichromic Heidenhain) and to perform further histochemical investigations (ie, GleesMarsland for neurons and neurofibrils, Bielschowsky for axons and dendrites, P.T.A.H. Mallory for glia) and immunohistochemical tests (to study apoptosis, various neuroreceptive structures, the expression of specific genes, etc), groups of 12 sections are performed, 3 of which are used for the histologic methods; the other 9 are saved and stained as deemed necessary for further investigations. Therefore, the number of groups of 12 serial sections varies from 30 in the fetus at the 25th week of gestation (correspondent to 360 sections) to 120 in SIDS victims over 6 months of life (correspondent to 1440 sections). Therefore, for each case analyzed, and according to the age of the subject, between 90 and 360 sections are stained with hematoxylin/eosin, KlüverBarrera, and trichromic Heidenhain, and between 270 and 1080 of unstained sections are kept and stained as necessary. This methodology takes a histotechnician 4 weeks of work.

The procedure for the simplified examination of the brainstem, both for the fetus and the infant, requires a much lower number of sections. The cranial, middle, and caudal portions of the nuclei can be examined, and the technique is applicable in every laboratory. However, this method requires a careful, precise sampling.

The brainstem is divided into 3 blocks, as shown in Figures 1 and 2. The first, cranial block extends by the border between the medulla oblongata and pons up to the upper pole

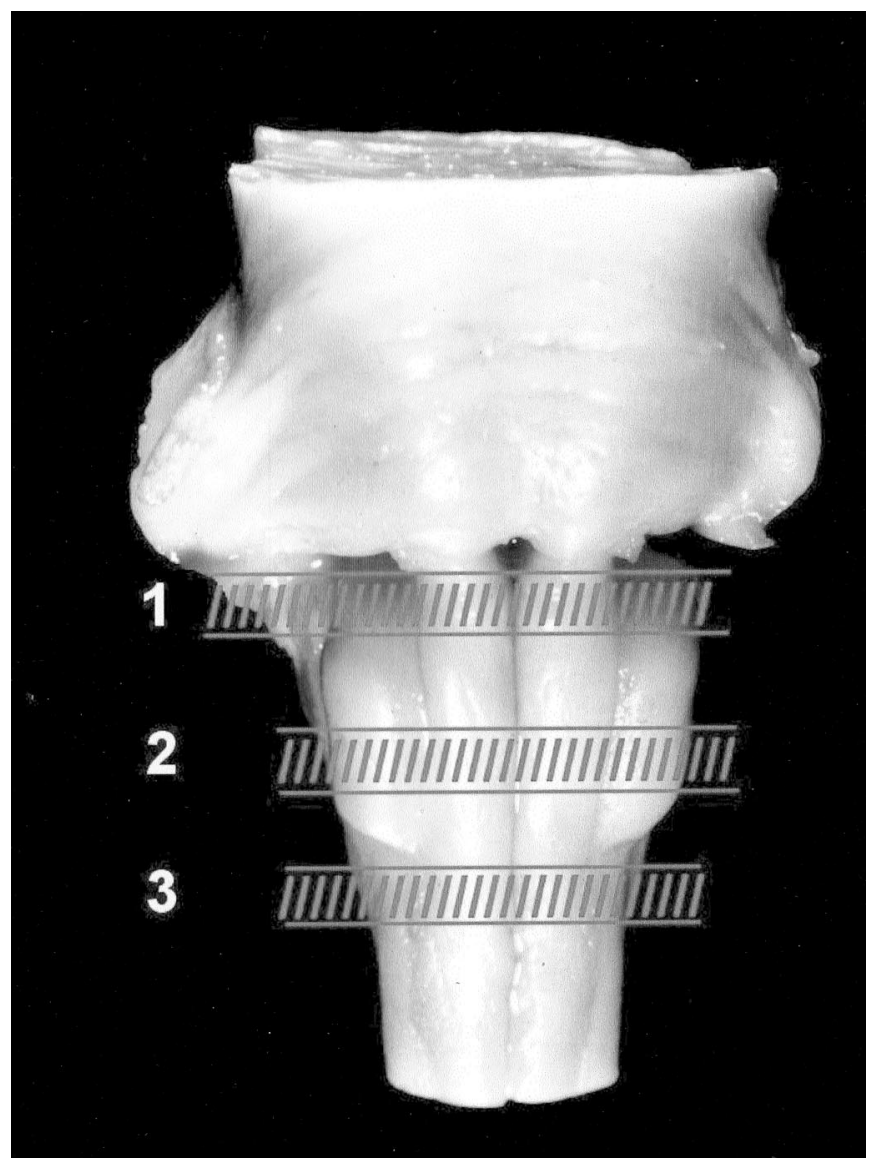

FIGURE 1. Brainstem: ventral surface. Numbers 1 to 3 indicate the blocks obtained by the subdivision of the medulla oblongata: 1, cranial block; 2, middle block; 3, caudal block. Diagonal lines indicate area to be sectioned. 


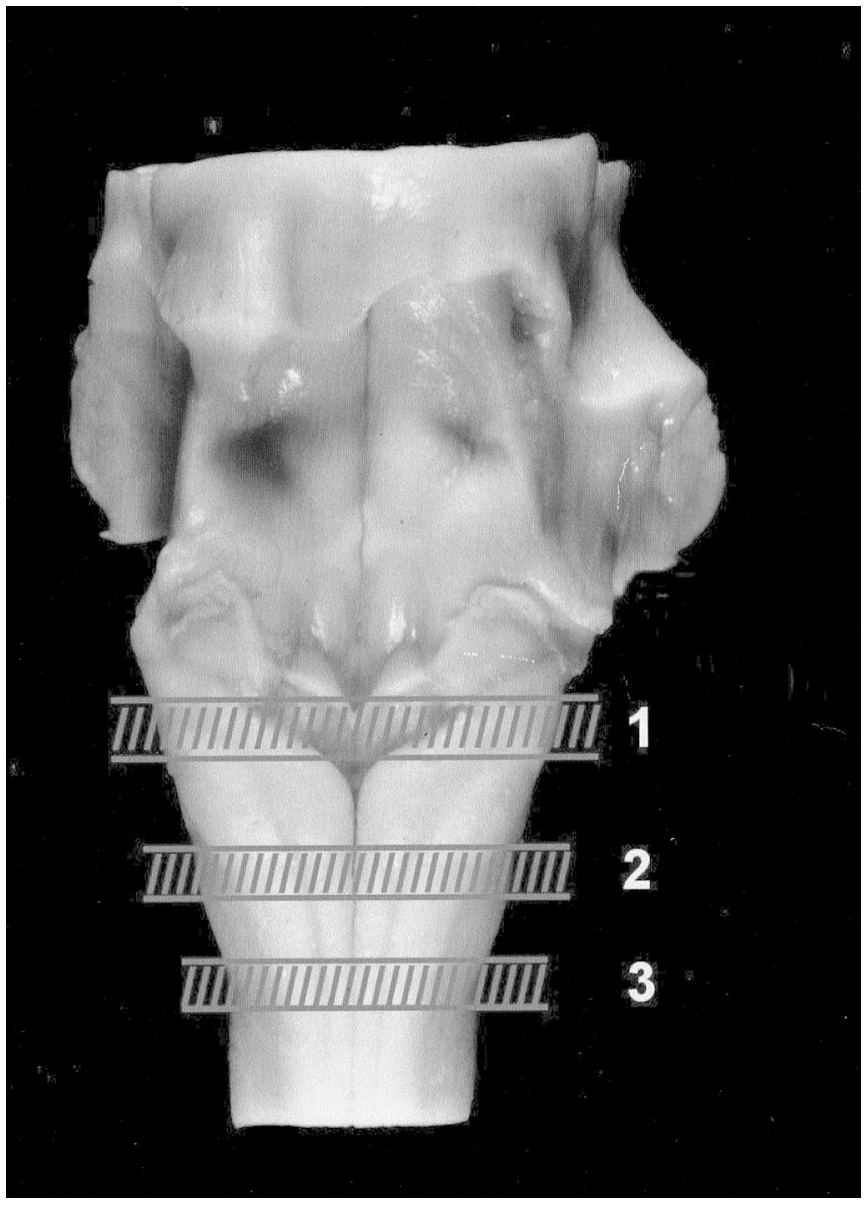

FIGURE 2. Brainstem: dorsal surface. Numbers 1 to 3 indicate the blocks obtained by the subdivision of the medulla oblongata: 1, cranial block; 2, middle block; 3, caudal block. Diagonal lines indicate area to be sectioned.

of the olivary eminence. The second, intermediate block, corresponding to the submedian area of the inferior olivary eminence, has as point of reference the obex and extends 2 to 3 millimeters above and below the obex itself. The third, caudal block, includes the lower pole of the inferior olivary eminence and the lower adjacent area of the medulla oblongata.

The first and second blocks, including respectively the upper third of the medulla oblongata with the adjacent portions of the pons and of the medulla oblongata adjacent to the obex, are sectioned in a cranial-caudal direction. The third block, corresponding to the lower portion of the medulla oblongata, is sectioned in a caudal-cranial direction. From each of these paraffin-embedded blocks, an average of 6 groups of 12 serial sections is obtained.

\section{RESULTS}

Examination of the brainstem in 106 SIDS victims, in 30 controls, and 51 SIUD victims results in a remarkable variability, particularly of the ARCn, both in size and neuronal density.

In the first cranial block, the ARCn is well recognizable, both medially and in the initial lateral portions. The point of reference is the border between the medulla oblongata and the pons, where the ARCn is well identifiable. At this level, the hypoglossus nucleus, the dorsal nucleus of the vagus, the tractus solitarius nucleus, and the ambiguous nucleus are just recognizable, while the reticular formation and the inferior olivary nucleus are evident.

In the second block, the ARCn appears clearly in the lateral portions, while it is reduced in the medial portion. The hypoglossus, dorsal of the vagus, ambiguus, inferior olivary nuclei, and the reticular formation are clearly detectable. The point of reference is the obex.

In the third block, the ARCn is detectable, particularly in the fetuses, in the lateral portions, while medially it is absent. All the other nuclei are well recognizable. The histologic point of repere reference is the area postrema. The borders of the ARCn are not always regular in comparison to the pyramidal bundles, being sometimes irregularly interspersed by the pyramidal bundles themselves.

Figure 3 shows the structural variability of the ARCn in 3 histologic sections from the medulla oblongata cranial (Fig. 3A), intermediate (Fig. 3B), and caudal block (Fig. 3C), respectively.

\section{DISCUSSION}

The identification of a possible pathologic basis of reflexogenic mechanisms in SIDS and SIUD requires examination of the nervous central system, central and peripheral, as well as of the cardiac conduction system. ${ }^{14}$ These structures are not routinely examined in traditional standardized necropsy procedures, including the "International Standardized Autopsy Protocol for Sudden Unexpected Infant Death," defined in 1996 by the members of National Institute of Child Health and Human Development. ${ }^{15}$

Recent studies have stressed the frequent alterations, mainly congenital in nature, of both the autonomic nervous system, central and peripheral, and the cardiac conduction system. ${ }^{11,12,16,17}$

Limiting the study of the autonomic nervous system to the brainstem, the congenital anomalies represented by dendritic spines, markers of neuronal immaturity, and most of all by hypoplasia of ARCn are of particular interest and frequency. ${ }^{8-12}$

Recent observations point out that such developmental alterations appear with a similar incidence in SIDS and SIUD victims. $^{3,11,12}$

ARCn hypoplasia is a congenital anomaly, not referable to any acquired alterations, and, particularly in SIUD, is associated with hypoplasia of other brainstem structures, particularly of the reticular respiratory formation. ${ }^{3}$ 

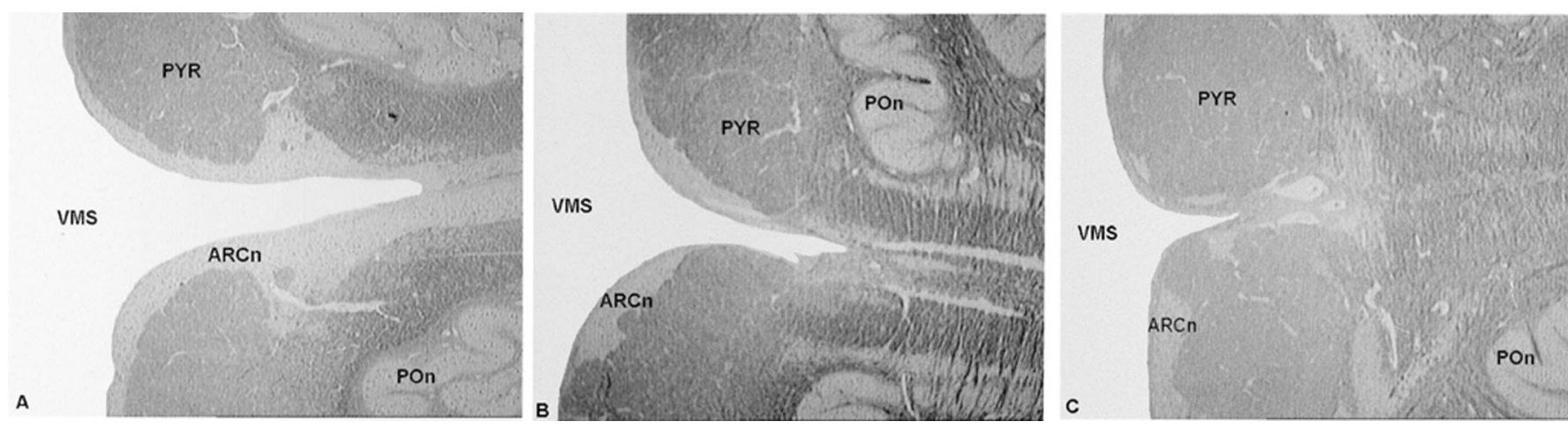

FIGURE 3. A, Section from the cranial medulla oblongata block with the arcuate nucleus predominantly located medially. B, Section from the intermediate medulla oblongata block with arcuate nucleus less evident in the median area. $\mathrm{C}$, Section from the caudal medulla oblongata block with the arcuate nucleus confined to lateral portions. Klüver-Barrera stains; $25 \times$. POn indicates principal inferior olive; PYR, pyramid; and VMS, ventral medullary surface.

The neuropathological study necessary for the objective identification of nature and location of each lesion, likely morphologic substrata of death, requires examination of a large number of cases according to homogeneous and standardized criteria. In this context, the unmodified frequency of SIUD in the last 20 years should be attributed to the scarce interest of the inherent anatomopathologic abnormalities. To this should be added the difficult evaluation of brainstem nuclei anomalies due to the lack of ontogenetic data in humans.

A complete brainstem examination on serial sections allows an objective dimensional and architectural evaluation of all the pertinent anatomic structures. Nevertheless, this study requires numerous sections and the systematic application of histologic and immunohistochemical techniques. Such complete brainstem examination takes a technician no less than 4 weeks.

The procedure for examination of the brainstem, on the other hand, allows evaluation of the different structures, and particularly of the ARCn, in all their extension, examining 3 specific levels, each one well defined by a morphologic reference point. Compared with the complete method, the simplified one requires a much lower number of the histologic slides. The 3 blocks are kept and sectioned as deemed necessary.

It should be underlined that, due to ARCn architectural variability, inaccurate indications of its structure and extension can occur upon examination of single, random sections. Moreover, a finding of ARCn absence could depend on separation of part of the ventral medullary surface due to tearing the leptomeninges during sampling.

Comparison of our observations on the ARCn with those reported in the classic anatomic textbooks by Testut, ${ }^{18}$ by Martin, ${ }^{19}$ and the Olszewski and Baxter atlas, ${ }^{20}$ yielded some agreements, and discordances have emerged.

Our description of the ARCn related to the upper brainstem portion corresponds as regards extension and lo- cation to the one reported by both Testut (ff 6, Fig. 203: brainstem section at the upper portion of the olive $)^{18}$ and Martin (Fig. AII-9: transverse section of the rostral brainstem). ${ }^{19}$ In the classic atlas by Olszewski and Baxter, ${ }^{20}$ in the figures corresponding to the levels 1601 to 1801 , the ARCn is located only at the limit between the medulla oblongata and the pons (level 1601), while in the immediate lower levels, corresponding to the upper pole of the olive, it is described as very poorly developed.

Regarding the intermediate level, our observations are in agreement with those by the other authors (level 2051 of the atlas, AII- 8 of Martin ${ }^{18}$ and ee-5 of Testut ${ }^{19}$ ).

Concordance is also achieved in our description of the $\mathrm{ARCn}$ at the lower level with that in the 2 texts (AII-7 of Martin $^{18}$ and dd-4 of Testut ${ }^{19}$ ), while in the atlas ${ }^{20}$ (level 2301), the nucleus is basically not mentioned.

On the basis of these considerations, we conclude that the autopsy protocol for SIDS victims, already internationally approved, ${ }^{15}$ must include examination of the autonomic nervous system according to the above-described guidelines. These will allow an accurate and standardized study of the brainstem, in addition to the examination of cardiac conduction system. ${ }^{16}$

The concept that SIDS is a form of death that remains unexplained after a complete autopsy should be revised. We suggest replacing the present SIDS definition by "the sudden death of an infant under one year of age which remains unexplained after a thorough case investigation, including performance of a complete autopsy and an in-depth histopathological analysis of the brainstem, examination of the death scene, and review of the clinical history."21

\section{ACKNOWLEDGMENTS}

This study was supported by the Lombardy Region (project 49210-24.3.00: "Program of research and intervention for the reduction of the risk of sudden infant death and 
unexpected fetal death") and MURST Cofin 2000 (project MM06153578: "Pathologic study of late fetal unexplained death-stillbirth"). The authors wish to express their gratitude to Simone G. Ramos, MD, and Gianmario Ballabio, MD, for advice and assistance with photographic prints.

\section{REFERENCES}

1. Willinger M, James S, Catz C. Defining the sudden infant death syndrome (SIDS): deliberations of an expert panel convened by the National Institute of Child Health and Human Development. Pediatr Pathol. 1991;11:127-132.

2. Moon RY, Omron R. Determinants of infant sleep position in an urban population. Clin Pediatr. 2002;41:569-573

3. Matturri L, Lavezzi AM, Minoli I, et al. Association between pulmonary hypoplasia and hypoplasia of arcuate nucleus in stillbirth. $J$ Perinatol. 2003;23:328-332.

4. Rossi L, Matturri L. Anatomohistological features of the heart's conduction system and innervation in SIDS. In: Rognum TO, ed. Sudden Infant Death Syndrome: New Trends in the Nineties. Oslo: Scandinavian University Press; 1995:207-212

5. Kalousek DK, Gilbert-Barness E. Causes of stillbirth and neonatal death. In: Gilbert-Barness E, ed. Potter's Pathology of the Fetus and Infant. St. Louis, Mo: Mosby; 1997:128-162.

6. Cotzias CS, Paterson-Brown S, Fisk NK. Prospective risk of unexplained stillbirth in singleton pregnancies at term: population based analysis. Br Heart J. 1999;319:287-288.

7. Kinney HC, Filiano JJ, Sleper LA, et al. Decreased muscarinic receptor binding in the arcuate nucleus in sudden infant death syndrome. Science. 1995;269:446-1450.

8. Kinney HC, Filiano JJ, Panigraphy A, et al. Anatomic and neurochemical studies of the ventral medulla oblongata in early life: observation relevant to the sudden infant death syndrome. In: Trouth CO, Hillis RH, Kiwull-Schone H, et al, eds. Ventral Brain Stem Mechanisms and Control Respiration and Blood Pressure. New York: Dekker; 1995: $589-609$.
9. Filiano JJ, Kinney HC. Arcuate nucleus hypoplasia in the sudden infant death syndrome. J Neuropathol Exp Neurol. 1992;51:394-403.

10. Matturri L, Biondo B, Mercurio P, et al. Severe hypoplasia of medullary arcuate nucleus: quantitative analysis in sudden infant death syndrome. Acta Neuropathol. 2000;99:371-375.

11. Matturri L, Minoli I, Lavezzi AM, et al. Hypoplasia of medullary arcuate nucleus in unexpected late fetal death (stillborn infants): a pathologic study. Pediatrics. 2002;109:E43.

12. Matturri L, Biondo B, Suàrez-Mier MP, et al. Brainstem lesions in the sudden infant death syndrome: variability in the hypoplasia of the arcuate nucleus. Acta Neuropathol. 2002;104:12-20.

13. Matturri L, Lavezzi AM, Ottaviani G, et al. Anatomo-pathological techniques for the study of brainstem in sudden infant death syndrome (SIDS) and unexpected late fetal stillbirth. Proceedings of the $7^{\text {th }}$ SIDS International Conference, Florence, Italy, 2002:131-132.

14. Rossi L, Matturri L. Cardiac conduction and nervous system in health, disease and sudden death: an anatomoclinical overview. Osp Maggiore. 1995;89:239-257.

15. Krous H. Instruction and reference manual for the international standardized autopsy protocol for sudden unexpected infant death. $J$ SIDS Infant Mortal. 1996;1:203-246.

16. Matturri L, Ottaviani G, Ramos S, et al. Sudden infant death syndrome (SIDS): a study of cardiac conduction system. Cardiovasc Pathol. 2000;9:137-145.

17. Ottaviani G, Matturri L, Rossi L, et al. Crib death: further support for the concept of fatal cardiac electrical instability as the final common pathway. Int J Cardiol. 2003;S2:17-26.

18. Testut L. Anatomia Umana: Libro Quinto: Sistema Nervoso Centrale. Torinese, Italy: Unione Tipografico Editrice; 1943.

19. Martin JH. Neuroanatomy: Text and Atlas. New York: Prentice Hall International; 1996.

20. Olszewski J, Baxter D. Cytoarchitecture of the Human Brain Stem. Basel, Switzerland: Karger; 1982.

21. Matturri L, Lavezzi AM, Rossi L. Proposal to modify the definition of SIDS, with regard to the post mortem exam. In: Proceedings of the 7th SIDS International Conference, Florence, Italy, 2002:103 